\title{
OPEN An ACE2 Triple Decoy that neutralizes SARS-CoV-2 shows enhanced affinity for virus variants
}

\author{
Shiho Tanaka ${ }^{1,7 凶}$, Gard Nelson ${ }^{1,7}$, C. Anders Olson ${ }^{1}$, Oleksandr Buzko ${ }^{1}$, Wendy Higashide ${ }^{1}$, \\ Annie Shin ${ }^{1}$, Marcos Gonzalez ${ }^{1}$, Justin Taft ${ }^{2,3,4}$, Roosheel Patel ${ }^{2,5}$, Sofija Buta ${ }^{2,5,6}$, \\ Ashley Richardson ${ }^{2,3,4,5,6}$, Dusan Bogunovic ${ }^{2,3,4,5,6}$, Patricia Spilman ${ }^{1}$, Kayvan Niazi ${ }^{1}$, \\ Shahrooz Rabizadeh ${ }^{1}$ \& Patrick Soon-Shiong ${ }^{1}$
}

The SARS-CoV-2 variants replacing the first wave strain pose an increased threat by their potential ability to escape pre-existing humoral protection. An angiotensin converting enzyme 2 (ACE2) decoy that competes with endogenous ACE2 for binding of the SARS-CoV-2 spike receptor binding domain (S RBD) and inhibits infection may offer a therapeutic option with sustained efficacy against variants. Here, we used Molecular Dynamics (MD) simulation to predict ACE2 sequence substitutions that might increase its affinity for S RBD and screened candidate ACE2 decoys in vitro. The lead ACE2(T27Y) $H 34 A$ )-Ig $G_{1} F_{C}$ fusion protein with enhanced $S$ RBD affinity shows greater live SARS-CoV-2 virus neutralization capability than wild type ACE2. MD simulation was used to predict the effects of $S$ RBD variant mutations on decoy affinity that was then confirmed by testing of an ACE2 Triple Decoy that included an additional enzyme activity-deactivating $\mathrm{H} 374 \mathrm{~N}$ substitution against mutated S RBD. The ACE2 Triple Decoy maintains high affinity for mutated S RBD, displays enhanced affinity for $S$ RBD N501Y or L452R, and has the highest affinity for S RBD with both E484K and N501Y mutations, making it a viable therapeutic option for the prevention or treatment of SARS-CoV-2 infection with a high likelihood of efficacy against variants.

SARS-CoV-2 variants have rapidly swept the globe ${ }^{1-3}$ and very recent investigations reveal that several of these variants have shown the ability to escape neutralization by convalescent antibodies in recovered COVID-19 patients ${ }^{4-10}$ and recombinant neutralizing antibodies (nAbs) developed as therapeutics ${ }^{11,12}$. There are also fears that current vaccines may not be as effective against some of the variants and early evidence suggests that for some vaccines, this risk may exist ${ }^{13,14}$. The latter is a particular concern, as the massive vaccine efforts currently underway employ vaccines designed to elicit immune responses against first-wave sequence SARS-CoV-2 spike (S) protein and specifically the $S$ receptor binding domain (S RBD) that binds to angiotensin-converting enzyme 2 (ACE2) on the surface of human cells in the airway and gut that initiates viral entry and infection ${ }^{15-18}$. While one response to the threat of loss of vaccine efficacy might be to continually re-design vaccines to target specific new variants, this would be an ongoing game of catch-up because it can be expected that further novel variants will emerge, particularly since several recent reports have shown that antibodies elicited by infection and vaccination act as evolutionary forces that result in the predominance of viral variants that escape these immune defenses ${ }^{19,20}$.

While efforts to adapt vaccines should be encouraged, in parallel, new therapeutic approaches to neutralize viral infection that are not undermined by the presence of mutations should be advanced.

To address the need for a therapeutic and potentially prophylactic approach that has a low likelihood of being adversely affected by variant mutations, we have designed and tested ACE2 'decoys' that leverage the binding of the S RBD to ACE2. This is an approach that is also being pursued by others using a variety of fusion proteins and delivery methods ${ }^{21-24}$. Our ACE2 decoys under development are recombinant $A C E 2-I_{1} G_{1} F_{C}$ or $-\operatorname{IgAF}_{C}$

${ }^{1}$ ImmunityBio, Inc., 9920 Jefferson Blvd., Culver City, CA 90232, USA. ${ }^{2}$ Center for Inborn Errors of Immunity, Icahn School of Medicine at Mount Sinai, 1 Gustave Lane, Levy Place, New York, NY 10029-5674, USA. ${ }^{3}$ Mindich Child Health and Development Institute, Icahn School of Medicine at Mount Sinai, 1 Gustave Lane, Levy Place, New York, NY 10029-5674, USA. "Department of Microbiology, Icahn School of Medicine at Mount Sinai, 1 Gustave Lane, Levy Place, New York, NY 10029-5674, USA. ${ }^{5}$ Department of Pediatrics, Icahn School of Medicine at Mount Sinai, 1 Gustave Lane, Levy Place, New York, NY 10029-5674, USA. ${ }^{6}$ Precision Immunology Institute, Icahn School of Medicine at Mount Sinai, 1 Gustave Lane, Levy Place, New York, NY 10029-5674, USA. ${ }^{7}$ These authors contributed equally: Shiho Tanaka and Gard Nelson. ${ }^{\square}$ email: Shiho.tanaka@immunitybio.com 
a

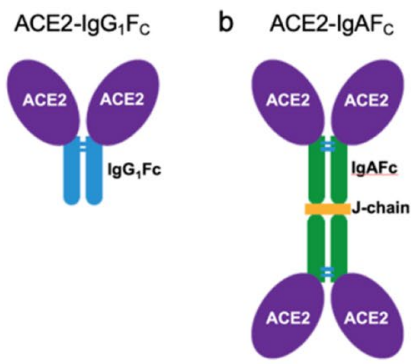

f $\quad$ ACE2(T27Y)- $-\operatorname{lgG}_{1} F_{\mathrm{C}}$

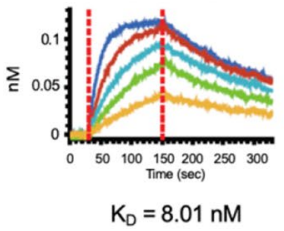

g

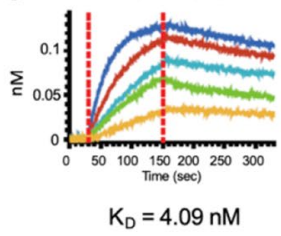

C
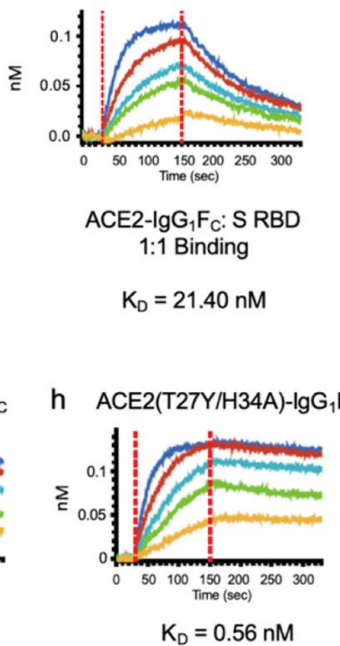

d

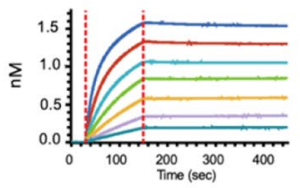

ACE2- $\lg G_{1} F_{C}: S$ RBD with avidity

$\mathrm{K}_{\mathrm{D}}=0.762 \mathrm{nM}$ e

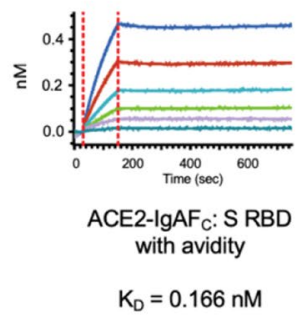

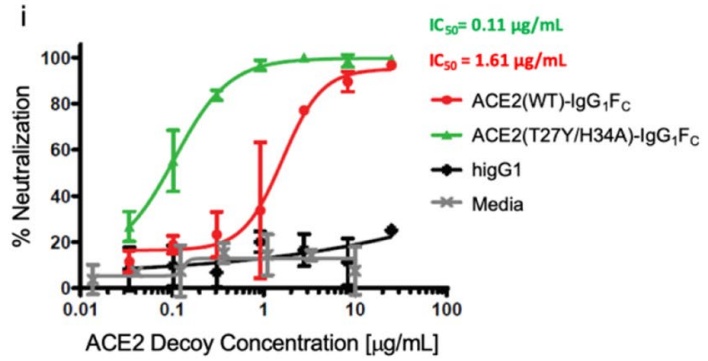

Figure 1. Biolayer Interferometry (BLI) of $A C E 2-\operatorname{IgG}_{1} F_{C}, A C E 2-\operatorname{IgAF} F_{C}$, and mutant decoy binding to the spike receptor binding domain; and live virus neutralization. The (a) ACE2-IgG $\mathrm{F}_{\mathrm{C}}$ decoy and (b) dimeric ACE2-IgAF $F_{C}$ decoy fused via a J-chain are shown. BLI kinetics analysis of (c) 1:1 binding and (d) binding with avidity for the ACE2-IgG $\mathrm{F}_{\mathrm{C}}$ decoy; (e) BLI binding with avidity for the ACE2-IgAF $\mathrm{F}_{\mathrm{C}}$ decoy; (f) ACE2(T27Y)$\mathrm{IgG}_{1} \mathrm{~F}_{\mathrm{C}},(\mathbf{g}) \mathrm{ACE} 2(\mathrm{H} 34 \mathrm{~A})-\mathrm{IgG}_{1} \mathrm{~F}_{\mathrm{C}}$, and (h) ACE2(T27Y/H34A)- $\operatorname{IgG}_{1} \mathrm{~F}_{\mathrm{C}}$ decoys are shown with $\mathrm{K}_{\mathrm{D}}$ values. (i) Virus neutralization as percent for concentrations of decoy is shown with IC50s for ACE2(WT)- $\operatorname{IgG}_{1} \mathrm{~F}_{\mathrm{C}}$ and ACE2(T27Y/H34A)-IgG $\mathrm{F}_{\mathrm{C}}$. Negative controls: media and higG1 (human IgG1).

fusion proteins, with the ACE2 sequence optimized for binding affinity to S RBD. The ACE2 decoy would be given to a patient infected with SARS-CoV-2, act to prevent binding of virus to host cell ACE2 by competing with endogenous ACE2 for spike binding, and allow clearance of the virus ${ }^{25-27}$.

To successfully compete, an efficacious ACE2 decoy would ideally have significantly higher affinity for S RBD than endogenous, host-cell expressed ACE2. To identify ACE2 mutations with a high probability of increasing affinity, we utilized our in silico Molecular Dynamics (MD) simulation capabilities as described in Nelson et al. ${ }^{28}$ "Millisecond-scale molecular dynamics simulation of spike RBD structure reveals evolutionary adaption of SARS$\mathrm{CoV}-2$ to stably bind ACE2" wherein we reported on our identification of regions of high affinity interaction between ACE2 and S RBD based on previously reported S RBD structures ${ }^{29,30}$.

Because the ACE2 decoy concept is based on interaction of ACE2 with S RBD, its binding affinity and thus efficacy may also be vulnerable to changes in the SARS-CoV-2 S RBD sequence. We therefore assessed the affinity of our ACE2 decoy, as compared to wild type (WT) ACE2, for S RBD with a variety of single or multiple mutations associated with the currently predominant variants, including the B.1.351 variant expressing E484K, $\mathrm{K} 417 \mathrm{~N}$, and N501Y mutations ${ }^{31}$, the B.1.1.7 variant (N501Y) ${ }^{1,32}$, and the B.1.427/B.1.429 L452R variant ${ }^{33-35}$.

Here, we report our findings that the combined T27Y and H34A mutations of ACE2 conferred the greatest increase in affinity for S RBD of the ACE2 variants tested. Our final ACE2 Triple Decoy also included an H374N mutation to abrogate ACE2 enzymatic activity. This ACE2 Triple Decoy not only maintained affinity for variant $\mathrm{S} R B D$, it showed an increased affinity for S RBD expressing N501Y or L452R mutations.

\section{Results}

Wild type (WT) ACE2-IgG $F_{C}$ and $A C E 2-\lg A F_{C}$ decoys show high affinity for $S$ RBD. In initial studies to design an ACE2 decoy, we determined the affinity of both recombinant wild type (WT) ACE2(WT)$\mathrm{IgG}_{1} \mathrm{~F}_{\mathrm{C}}$ and $-\mathrm{IgAF}_{\mathrm{C}}$ fusion proteins for binding to $\mathrm{S}$ RBD by Biolayer Interferometry (BLI) analysis. The ACE2(WT)-IgG $\mathrm{F}_{\mathrm{C}}$ decoy (Fig. 1a) showed moderate affinity for S RBD in 1:1 binding with a coefficient of dissociation $\left(\mathrm{K}_{\mathrm{D}}\right)$ of $21.40 \mathrm{nM}$ and high binding with avidity with a $\mathrm{K}_{\mathrm{D}}$ of $0.762 \mathrm{nM}$ (Fig. 1c,d, respectively). The ACE2(WT)-IgAF $F_{C}$ dimeric fusion protein (Fig. 1b) demonstrated even higher binding (with avidity) affinity for $\mathrm{S}$ RBD with a $\mathrm{K}_{\mathrm{D}}$ of $0.166 \mathrm{nM}$ (Fig. 1e). Additional BLI kinetics values are shown in Table S1 in the "Supplementary Information S1".

An ACE2 decoy expressing T27Y and H34A mutations shows enhanced affinity for S RBD and improved neutralization of live SARS-CoV-2. Based on MD simulation-based predictions of mutations that may confer enhanced binding affinity of ACE2 for S RBD, several ACE2 variants were tested for binding affinity as $A C E 2-\operatorname{IgG}_{1} F_{C}$ fusion proteins. A tyrosine $(Y)$ substitution for threonine $(\mathrm{T})$ at residue 27 (Fig. 1f) and an alanine (A) substitution for histidine $(\mathrm{H})$ at residue 34 (Fig. 1g) of ACE2 resulted in 3-5-fold increases in binding affinities (T27Y $\mathrm{K}_{\mathrm{D}}=8.01 \mathrm{nM} ; \mathrm{H} 34 \mathrm{~A} \mathrm{~K} \mathrm{D}_{\mathrm{D}}=4.09 \mathrm{nM}$ ). Combination of the T27Y and H34A substitutions 
a

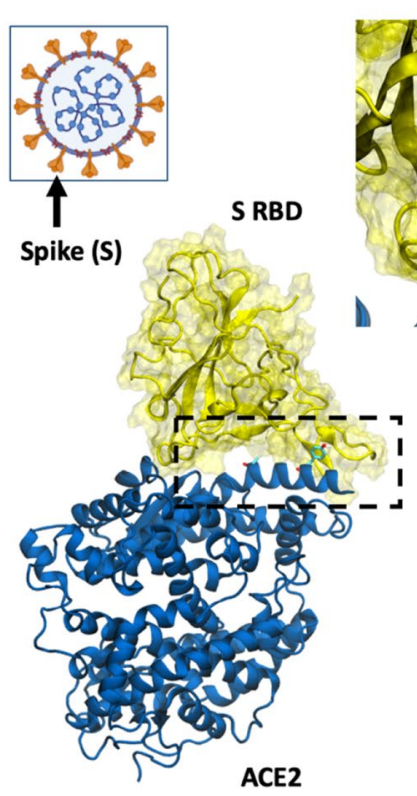

b

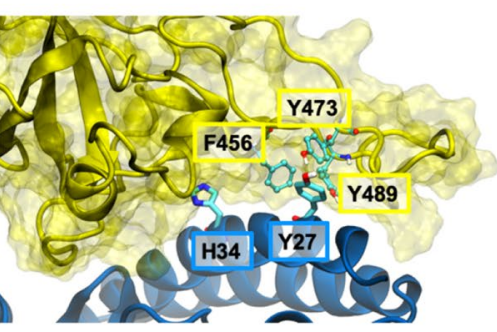

C

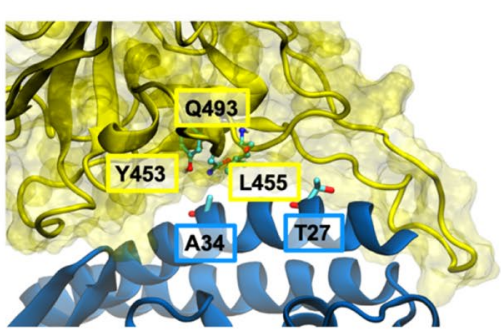

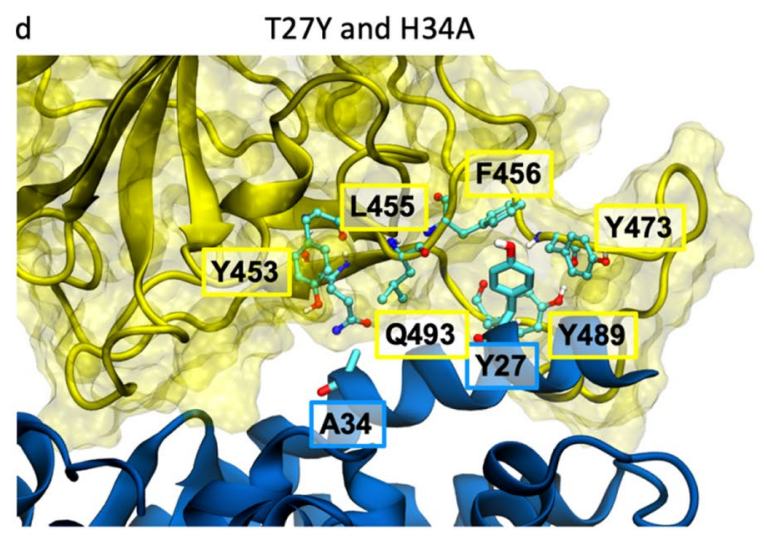

Figure 2. Molecular effects of T27Y and H34A ACE2 mutations predicted by MD simulation. (a) Spike (S) occurs as a trimer on the viral surface (orange projections), with the receptor binding domain (RBD) being on the outermost surface. The interface between S RBD and ACE2 is within the dashed box. Simulation models are shown for (b) ACE2(T27Y)-, (c) ACE2(H34A)-, and (d) ACE2(T27Y/H34A)-S RBD interactions. S RBD residues are labeled in the yellow boxes and ACE2 residues in blue boxes.

gave synergistic enhancement of binding affinity, showing an $~ 35$-fold increase in binding affinity as compared to ACE2(WT) with the $\mathrm{K}_{\mathrm{D}}$ decreasing to $0.56 \mathrm{nM}$ (Fig. 1h).

The ACE2(T27Y/H34A)- $\mathrm{IgG}_{1} \mathrm{~F}_{\mathrm{C}}$ double decoy was compared to the ACE2(WT)-IgG $\mathrm{F}_{\mathrm{C}}$ decoy in a live SARSCoV-2 virus assay using Vero E6 cells. The double mutant ACE2 decoy showed a 15-fold improvement in SARSCoV-2 neutralization capability compared to the ACE2(WT) decoy (Fig. 1i). Additional BLI kinetics values and $\mathrm{IC}_{50}$ in the live virus assay are shown in Table S2 in the "Supplementary Information S1".

MD simulations provide insight into the greater affinity of ACE2 T27Y and H34A for S RBD. MD simulations of interactions at the S RBD:ACE2 interface (Fig. 2a) suggest that for the ACE2 T27Y and $\mathrm{H} 34 \mathrm{~A}$ substitutions, the tyrosine substitution for threonine at residue 27 (Fig. 2b) introduces favorable hydrophobic contacts with RBD. The alanine substitution for histidine at residue 34 of ACE2 (Fig. 2c) allows more surface area for RBD residues to contact the ACE2 helix and may favorably increase entropy by increasing side chain flexibility, as evidenced by the root-mean-square deviation (RMSD) of RBD residues in contact with ACE2 residue 34, which increases from $1.8 \AA$ for $\mathrm{H} 34$ to $2.1 \AA$ for A34. Synergy between these mutations (Fig. 2d) occurs since their effects are independent and do not perturb the binding pose.

Addition of an H374N mutation inhibits ACE2 enzyme activity. In addition to enhanced affinity for competitive binding of S RBD, we wanted to inhibit the enzymatic activity of ACE2 ${ }^{36}$. Angiotensin-converting enzyme 2 has an important role in homeostasis of the renin-angiotensin system ${ }^{37-39}$ by cleavage of its substrate angiotensin $1-9^{40}$ and its activity affects a variety of systems. Addition of enzymatically active recombinant ACE2 to the system presents a high risk of unwanted side effects and since S RBD binding, but not substrate cleavage activity, is the key function for the ACE2 decoy, we tested a variety of mutations predicted to inhibit ACE2 enzymatic activity with a low likelihood of affecting S RBD binding affinity.

All of the ACE2 mutations (R273Q, R273K, R273L, H345A, H505L, H374N, or H378N) predicted or known to inhibit ACE2 enzymatic activity ${ }^{41,42}$ did inhibit this activity in the assay ("Methods" and Fig. S1 in the "Supplementary Information S1").

ACE2 triple mutant decoys comprising the S RBD binding affinity-enhancing T27Y/H34A mutations and the enzymatic activity-inhibiting mutations were produced and binding affinity assessed. Of the triple mutants, those with either the R273K or H374N mutations showed the highest S RBD affinity (Table S3 in the "Supplementary Information S1").

The final ACE2 Triple Decoy chosen for further testing was ACE2 (T27Y/H34A/H374N)- IgG $_{1} \mathrm{~F}_{\mathrm{C}}$ due to its more favorable biophysical characteristics as compared to the R273K-containing triple mutant, including a lower propensity to aggregate and a higher titer (Fig. S2 and Table S4 in the "Supplementary Information S1"). 


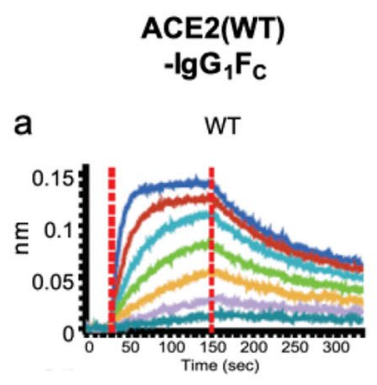

ACE2(T27Y/H34A/H374N) $-\lg \mathbf{G}_{1} \mathbf{F}_{\mathrm{C}}$

b

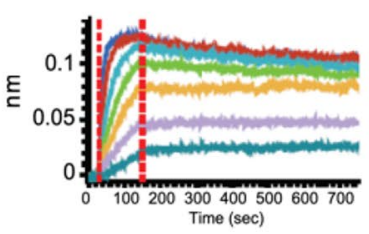

e

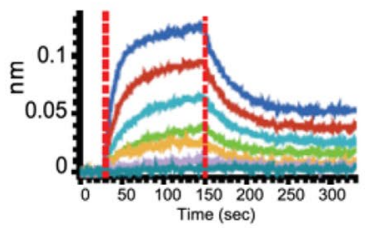

i

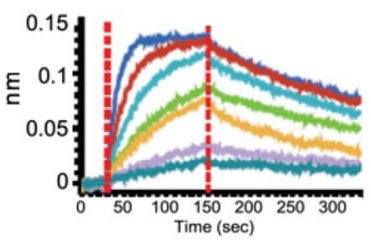

m

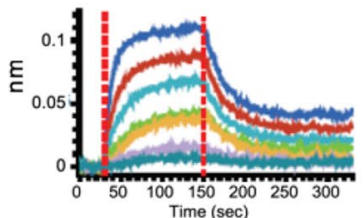

q

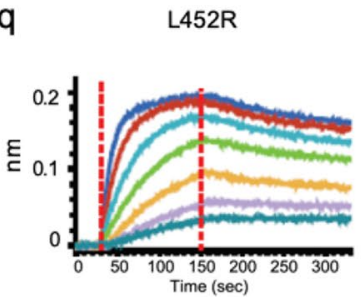

f

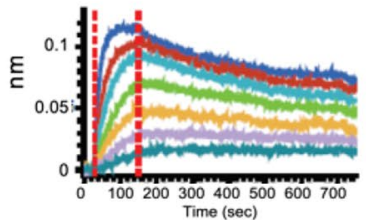

j

$\mathrm{n}$

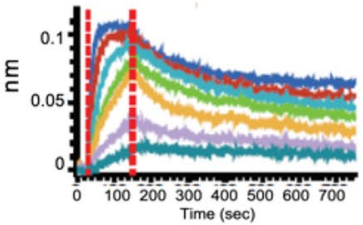

r

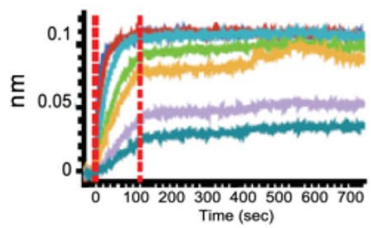

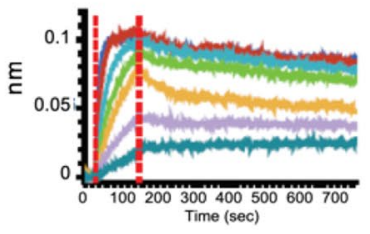

ACE2(WT)

$-\lg G_{1} F_{C}$

C

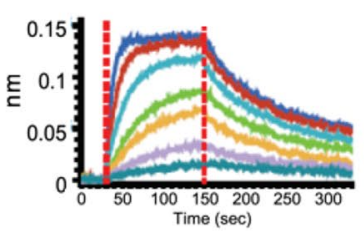

g

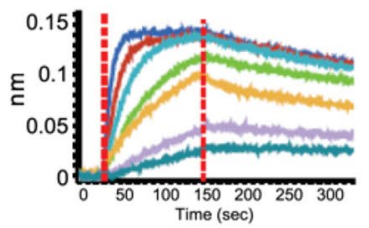

k

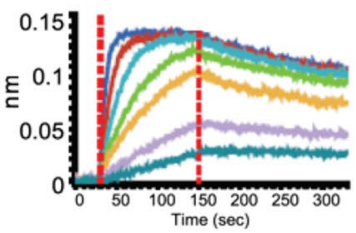

o

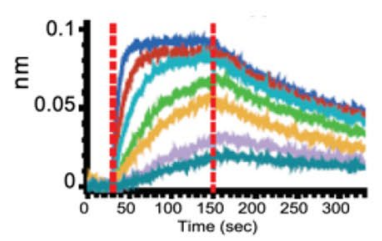

p K417/E484K/N501Y

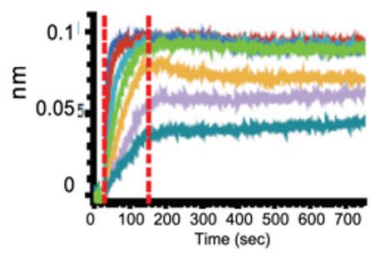

ACE2 Triple Decoy with

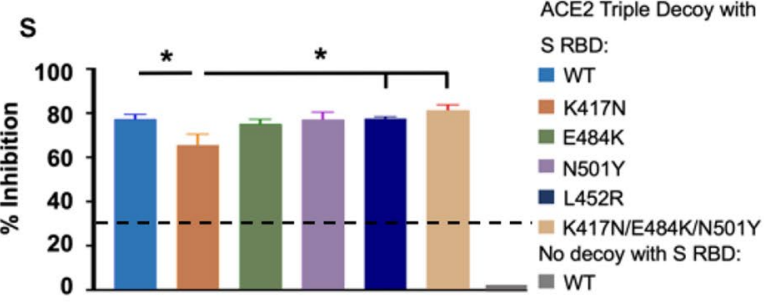

Figure 3. BLI of ACE2(WT) or Triple Decoy for mutated RBD and inhibition in the neutralization assay. (a-r) BLI of WT or Triple Decoy ACE2 to WT or mutated S RBD are shown side-by-side. (s) The percent inhibition of RBD binding to ACE2 in the surrogate neutralization assay is shown for the ACE2 Triple Decoy with S RBD $\mathrm{WT}$ and listed variants. RBD concentrations were $25 \mu \mathrm{g} / \mathrm{mL}$. The negative control is no decoy. Inhibition of $\geq 30 \%$ (dashed line) correlates with neutralization of the virus. Data graphed as mean with SEM. Statistics: oneway ANOVA and Tukey's post-hoc analysis comparing Triple Decoy binding to RBD WT/variants. For RBD K417N vs WT, $p=0.0495$; vs L452R, $\mathrm{p}=0.0451$; and vs $\mathrm{E} 484 \mathrm{~K} / \mathrm{K} 417 \mathrm{~N} / \mathrm{N} 501 \mathrm{Y}, \mathrm{p}=0.0128$.

The ACE2 Triple Decoy shows enhanced binding to S RBD N501Y and L452R variants, with the highest affinity for S RBD with both N501Y and E484K. The BLI analysis of both the ACE2(WT)$\mathrm{IgG}_{1} \mathrm{~F}_{\mathrm{C}}$ and the ACE2 Triple Decoy to $\mathrm{S}$ RBD WT or a S RBD with a series of mutations found in the B.1.351 ${ }^{43}$ (E484K/K417N/N501Y), B.1.1.7 (N501Y) ${ }^{1,32}$, and B.1.427/B.1.429 (L452R) ${ }^{33}$ variants is shown in Fig. 3 and the KD values in Table S5 in the "Supplementary Information S1".

The ACE2 Triple Decoy showed higher binding affinity to all S RBD sequences as compared to the ACE2(WT) decoy. As compared to the ACE2 Triple Decoy binding affinity for S RBD WT, affinities for S RBD E484K/N501Y, N501Y alone and L452R were higher; affinities for S RBD E484K, K417N/N501Y, N417N/E484K/N501Y, K417K/ 
E484K, and K417N were lower. Findings were similar with the wild type ACE2 decoy, with the highest affinity seen for E484K/N501Y and N501Y alone, and the lowest affinities for variants expressing K417N. N501Y and L452R showed 2-3-fold increase in binding affinity for both wild type ACE2 decoy and ACE2 Triple Decoy. E484K alone did not affect binding affinity to ACE2. K417N weakened binding affinity for ACE2(WT) and the Triple Decoy, but affinity was restored when combined with N501Y. The E484K, K417N and N501Y mutations occur together in the B.1.351 strain, whereas L452R alone is found in B.1.427/B.1.429, therefore assessment of ACE2 WT binding to these variants as they occur in nature may be considered the most physiologically relevant (Fig. S3 and Table S6 in the "Supplementary Information S1").

Given the rapidly evolving landscape of variants and the risk of recombination events, to extend our analysis to 'theoretical' variants, in this instance combination of L452R with other mutations, we produced and tested S RBD L452R/K417N, L452R/E484K, L452R/N501Y, and L452R/K417N/E484K/N501Y. All but the construct expressing L452R/K417N showed higher affinity for ACE(WT) (Table S7 in the "Supplementary Information S1"), which aligns with what might be predicted from the results in Table S5-K417N decreases affinity and N501Y increases affinity. Interestingly, affinity in combination appears to be additive, with the $\mathrm{K}_{\mathrm{D}}$ being 8.77 for E484K alone (as compared to 9.33 for RBD WT) and 5.58 for L452R alone; together the KD for binding to ACE2 (WT) is 4.10.

Inhibition of ACE2:S RBD binding in the surrogate virus neutralization assay correlates with binding affinity. The surrogate SARS-CoV-2 neutralization assay $\mathrm{cPass}^{\mathrm{m} 44}$ is based upon assessment of inhibition of binding of ACE2 (WT) to S RBD (WT). It is typically used to ascertain the presence of anti-S RBD antibodies in serum. Such antibodies inhibit binding of S RBD to ACE2 bound to an ELISA plate, and inhibition of $\geq 30 \%$ has been reported to correlate with neutralization of live virus. Here, the surrogate assay was used to determine if the ACE2 Triple Decoy could inhibit S RBD WT and variant binding to plate-bound ACE2, that is, compete with ACE2 (WT) for S RBD binding (see "Methods").

As shown in Fig. 3s, inhibition in the surrogate virus neutralization assay modified to assess competition by the ACE2 Triple Decoy for ACE2 (WT) binding to S RBD was similar for S RBD WT, E484K, N501Y, L452R, and K417N/E484K/N501Y. The percent inhibition with the ACE2 Triple Decoy and S RBD K417N was significantly lower than the other mutants, but still very high.

MD simulation accurately predicts the relative affinities confirmed by in vitro testing. To predict binding affinities, we used Adaptively-biased MD (ABMD) simulations ${ }^{45}$, which allow observation and quantification of binding and unbinding, of both ACE2 WT and ACE2 (T27Y/H34A) binding to S RBD WT or B.1.351. For these simulations, the B.1.351 variant comprising the $\mathrm{E} 484 \mathrm{~K}, \mathrm{~K} 417 \mathrm{~N}$, and $\mathrm{N} 501 \mathrm{Y}$ mutations was used because these mutations occur together naturally and thus this combination has high physiological relevance. Furthermore, this variant has been reported to escape antibodies elicited by first-wave SARS-CoV-2 and some vaccines, ${ }^{46-50}$ and is of particular interest. The ACE2 T27Y/H34A sequence without the additional H374N enzyme-deactivating mutation found in the ACE2 Triple Decoy was used because earlier simulations had been unable to detect a change in affinity due to the presence of the H374N mutation.

We predicted binding affinities by determining the Helmholtz binding free energy $\left(\Delta \mathrm{A}_{\text {bind }}\right)$ using the ratio of the probability of the bound and unbound states based on the Free Energy Surfaces (FES) (Fig. 4), where more negative values of $\Delta \mathrm{A}_{\text {bind }}$ indicate a stronger association. Details of the ABMD simulations and Helmholtz calculation can be found in "Methods". The calculated free energies of binding, in order of predicted affinity from lowest to highest, are: ACE2 WT:RBD WT ( $-4.06 \pm 0.06 \mathrm{kcal} / \mathrm{mol}$; Fig. 4a); ACE2 WT:RBD B.1.351 (-4.92 $\pm 0.14 \mathrm{kcal} /$ mol; Fig. 4b); ACE2 T27Y/H34A:RBD B.1.351 ( $-5.99 \pm 0.13 \mathrm{kcal} / \mathrm{mol}$; Fig. 4c); and ACE2 T27Y/H34A:RBD WT $(-6.85 \pm .13 \mathrm{kcal} / \mathrm{mol}$; Fig. $4 \mathrm{~d})$.

The predictive utility of these simulations is validated by the $\mathrm{K}_{\mathrm{D}}$ values determined in vitro and presented in Table S5 in the "Supplementary Information S1", where (for the combinations tested in MD simulations) the lowest affinity was also seen for ACE2 WT: RBD WT $\left(K_{D}=9.33 \mathrm{nM}\right)$, followed by ACE2 WT: RBD B.1.351 $\left(\mathrm{K}_{\mathrm{D}}=5.28 \mathrm{nM}\right)$, then ACE2 Decoy: RBD B.1.351 $(\mathrm{KD}=0.465 \mathrm{nM})$, and ACE2 Decoy: RBD WT $(\mathrm{KD}=0.315 \mathrm{nM})$. All affinities were high, and higher for Triple Decoy binding than ACE2 WT for all RBD sequences tested.

\section{Discussion}

To our knowledge, we are the first to report binding affinities of a recombinant mutant ACE2 decoy to the spike receptor binding domain expressing N501Y, E484K, N417Y, or L452R mutations; although we note Huang et al. reported previously on the affinity of their ACE2- $\mathrm{F}_{\mathrm{C}}$ to $\mathrm{S}$ RBD with the D614G mutation ${ }^{51}$. The greater affinity of ACE2 for S RBD with the N501Y substitution alone or in combination with E484K reported here is in alignment with our findings in Nelson et al. ${ }^{52}$, wherein we used MD simulation to predict that these mutations have a high probability of increasing affinity for ACE2.

The MD simulation data presented here was used to guide design of the ACE2 Triple Decoy and to predict affinities of the decoy as compared to ACE2 WT for a series of variants reveal again the merits of such simulations as a tool to inform therapeutic design.

Interestingly, widespread use of an ACE2 decoy has the potential itself to act as an evolutionary force; however, an ACE2 decoy largely recognizes the same residues as endogenous ACE2 and therefore it is highly unlikely a SARS-CoV-2 variant could emerge that 'escapes' the decoy yet still binds to endogenous ACE2. This phenomenon along with limited use of a decoy for therapy as compared to the spread of virus in a large population with opportunity for selection, makes the decoy approach less vulnerable to loss of efficacy due to mutation of the virus.

The enhanced binding affinity of our Triple Mutant ACE2 Decoy to S RBD with the variant mutations tested here supports continued pursuit of this therapeutic approach and further provides hope that even should the efficacy of vaccines currently in distribution or therapeutic neutralizing antibodies raised against WT spike be 
a

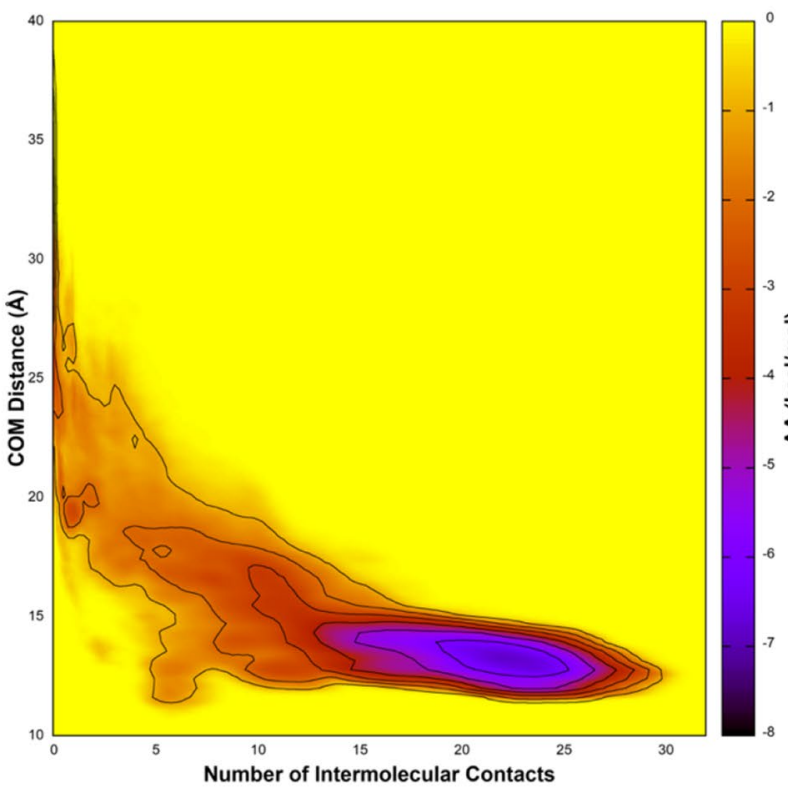

C

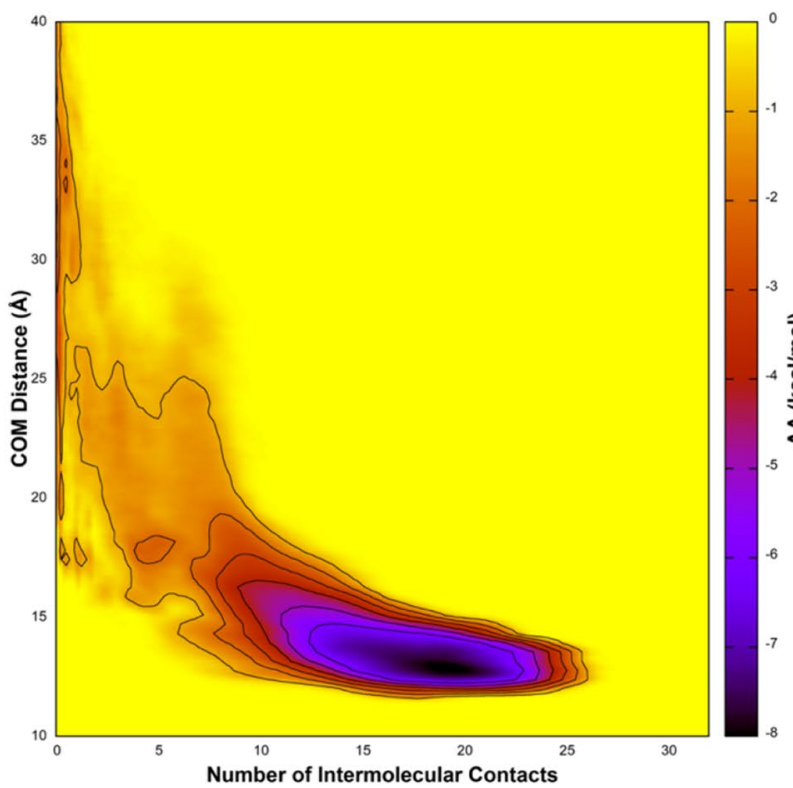

b
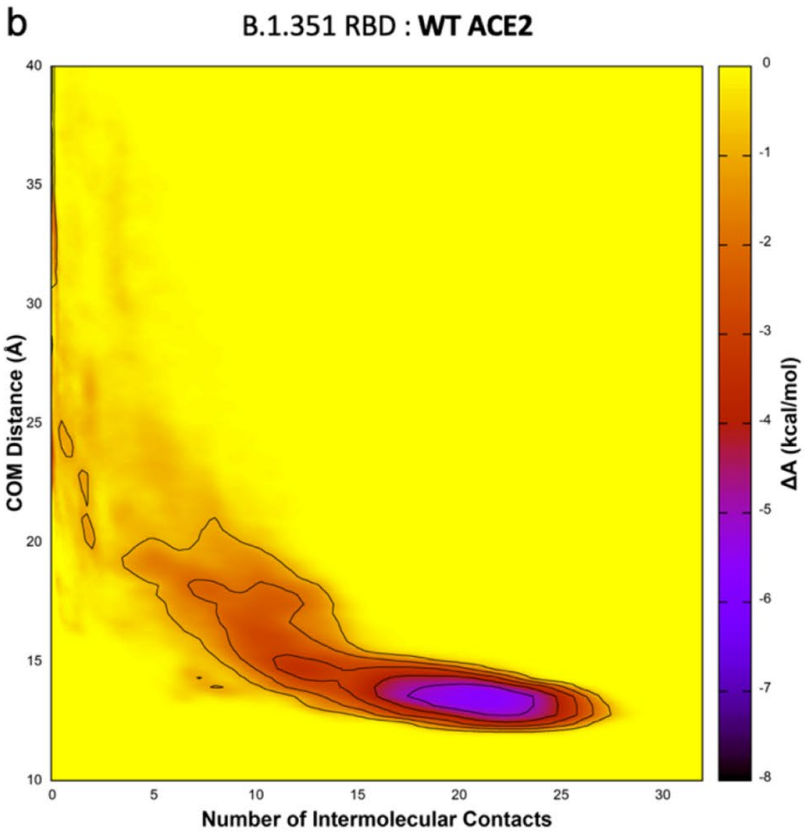

d

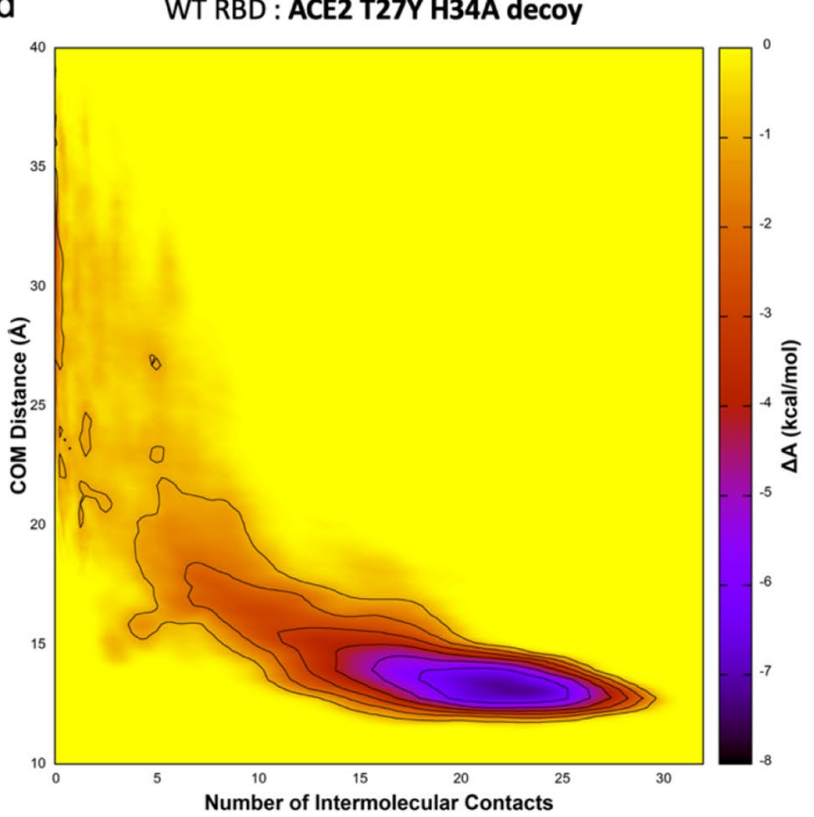

Figure 4. MD simulation predicts highest affinity for the T27Y/H34A decoy to S RBD WT and B.1.351. The free energy surfaces (FES) of wild type (WT) ACE2 upon interaction with (a) WT RBD or (b) B.1.351 RBD; and FES for the ACE2 T27Y/H34A decoy and (c) B.1.351 RBD or (d) WT RBD are shown. Darker purple represents lower free energy $\left(\Delta \mathrm{A}_{\mathrm{FES}}\right.$, scale at right of each panel). The free energy is a function of the number of intramolecular contacts (x-axis) and the distance between the centers of mass (COM, $y$-axis) of the interface regions. Binding free energy $\left(\Delta \mathrm{A}_{\text {bind }}\right)$ is estimated by integrating the FES using Eq. (1) in "Methods".

lessened by these variants, there will be an alternative therapeutic approach to successfully treat COVID-19 disease.

In our next steps in development of the ACE2 Triple Decoy, we will address the challenge of stability and successful delivery. Others developing ACE2 decoys have suggested use of intranasal ${ }^{53}$ or nanoparticle/extracellular vesicle delivery ${ }^{22,54-56}$. We anticipate going forward into our next studies using the dimeric IgA ${ }^{57}$ fusion protein decoy expressed by the human adenovirus serotype 5 E1, E2b, E3 deleted (hAd5 [E1-, E2b-, E3-]) platform that we have used successfully in our vaccine development ${ }^{58,59}$. This platform can readily be used to generate oral and/or intranasal formulations to further facilitate delivery. Our ACE2 Triple Decoy delivered in vivo using the hAd5 platform is anticipated to overcome barriers to successful delivery and will be tested in animal models of SARS-CoV-2 infection in future studies. 


\section{Methods}

MD simulation. System setup. The WT-ACE2/RBD complex was built from the cryo-EM structure, PDB $6 \mathrm{M} 17$ of full-length human ACE2 in the presence of the neutral amino acid transported $\mathrm{B}^{0} \mathrm{AT} 1$ with the S RBD as shown in Yan et al. ${ }^{60}$ using RBD residues 336-518 and ACE2 residues 21-614. ACE2 residues 27 and 34 were mutated to tyrosine and alanine, respectively. The final simulation system was built using the Amber ff $14 \mathrm{SB}$ force field ${ }^{61}$. The RISM program from AmberTools $19^{62}$ was used to determine optimal locations for water molecules in direct contact with the proteins. Bulk waters were added to create a sufficient octahedral water box and sodium ions were added at random locations to neutralize the system. The TIP3P force field was used for water and the Li/Merz parameters for sodium ${ }^{63}, 64$. After introducing mutations at the relevant residues, the same procedure was used to generate the other three systems.

Simulation. Ten copies of each RBD:ACE2 complex were minimized, equilibrated and simulated. Minimization occurred in two phases. During the first, the protein and RISM-placed waters were restrained. The second phase minimized the entire system. Dynamics then began and the temperature was ramped from 0 to $300 \mathrm{~K}$ while restraining the protein and RISM-placed waters. All dynamics used SHAKE restraints on hydrogen-containing bonds and a 2 fs timestep. All restraints were then released and the system was equilibrated in the NPT ensemble for $2 \mathrm{~ns}$. Finally, each system was equilibrated in the NVT ensemble for $100 \mathrm{~ns}$.

Steered MD was used to prepare the equilibrated systems for free energy calculation. Contacting residues from the adaptively biased MD (ABMD) simulations in Nelson et al. ${ }^{28,45}$ were used. Starting from the NVT equilibrated structures and over a 10 ns simulation, the number of intermolecular contacts was linearly reduced to 0 using a $10 \mathrm{kcal} / \mathrm{mol}^{\star} \AA$ A steering bias. Structures were randomly selected from the steered MD simulations and used to seed ABMD simulations. Two dimensional ABMD simulations used intermolecular contacts and the center of mass distance as collective variables. Centers of mass were defined as the alpha carbons from all interfacial residues in each molecule. The well-tempered ABMD bias potentia ${ }^{65}$ was used for free energy calculations. Our goal with the simulations was to inform the experiment by ranking the affinities of each complex instead of predicting the magnitude of the binding affinity. Based on our experience with the ACE2 WT:RBD WT complex ${ }^{28}$, we stopped the ABMD simulations after a total of $15.6 \mu \mathrm{s}, 16.0 \mu \mathrm{s}, 16.0 \mu \mathrm{s}$ and $16.0 \mu \mathrm{s}$ for the ACE2 WT:RBD WT, RBD WT:ACE2 T27Y/H34A, RBD B.1.351:ACE2 WT and RBD B.1.351:ACE2 T27Y/H34A, respectively. The time series showing full sampling of both CV s from the ACE2 WT:RBD WT simulation is shown in Figure S4 in the "Supplementary Information S1". It is representative of the other three systems in that we see full coverage of both CVs and multiple transitions between the bound and unbound states. Production simulations were run in the NVT ensemble meaning the calculated free energy corresponds to the Helmholz free energy $(\Delta \mathrm{A})$.

ABMD produces a free energy surface (FES) that describes the relative free energy between any two points on the FES, $\Delta \mathrm{A}_{\mathrm{FES}}$. The binding free energy $\left(\Delta \mathrm{A}_{\mathrm{bind}}\right)$ is determined by the ratio of the probability of the bound and unbound states and can be determined from the FES:

$$
\Delta A_{\text {bind }}=-\frac{1}{\beta} \ln \frac{\int_{\text {Bound }} d x d y e^{-\beta \Delta \mathrm{A}_{\mathrm{FES}}(x, y)}}{\int_{\text {Unbound }} d x d y e^{-\beta \Delta \mathrm{A}_{\mathrm{FES}}(x, y)}}
$$

where $\beta$ is the inverse of the Boltzmann constant multiplied by the temperature in Kelvins. More negative values of $\Delta \mathrm{A}_{\text {bind }}$ indicate a stronger association. The calculated $\Delta \mathrm{A}_{\text {bind }}$ values can be directly compared.

The "Bound" integral in Eq. (1) is defined to be over all $\Delta \mathrm{A}_{\mathrm{FES}}(\mathrm{x}, \mathrm{y})$ values with the number of contacts greater than 0.05 while the "Unbound" integral is over all values with fewer than 0.05 contacts. $\Delta \mathrm{A}_{\text {bind }}$ was calculated with different boundaries ranging from 0.0 to 1.0, inclusive. As expected, the resulting values of $\Delta \mathrm{A}_{\text {bind }}$ changed based on the chosen boundary. However, the relative ordering of the values did not. The value of 0.05 contacts was chosen as the boundary because it allowed for unambiguous categorization of points as either "unbound" $(\mathrm{x}=0)$ or "partially" or "fully bound". All simulations were performed with the GPU-enabled version of pmemd from Amber $20^{62}$. Multiple-walker ABMD simulations ${ }^{66}$ used the MPI version of pmemd.cuda from Amber20. For each system, average $\Delta \mathrm{A}$ and standard deviation values were calculated over the final ten percent of simulation data from each walker.

The RMSD for the RBD residues contacting ACE2 residue 34 were calculated from unbiased MD simulations. The ten 100 ns NVT WT RBD:WT ACE2 simulations were extended to a total of $5 \mu$ s. An A34 simulation system was set up and equilibrated following the procedure outlined above and $5 \mu$ s of unbiased NVT data was collected. The simulation trajectories were aligned to the alpha carbons of ACE2 residues 23-47. RMSD was then measured for heavy atoms of RBD residues 453, 455 and 493, which are in contact with residue H34 in the WT complex. Results were calculated using cpptraj ${ }^{67}$.

Production of ACE2 Decoys and S RBD. Expression constructs. Polymerase Chain Reactions (PCR) were conducted using PrimeSTAR GXL DNA Polymerase (Takara Bio) per the manufacturer's instructions. Primers and Gene Fragments were synthesized by Integrated DNA Technologies (IDT). For Gibson Assembly, NEBuilder Hifi DNA Assembly Master Mix (New England Biolabs) was used. For DNA ligation, we used T4 DNA Ligase (NEB) per the manufacturer's instructions. Plasmid sequences were confirmed by Sanger sequencing (Genewiz).

ACE2-IgG $\mathrm{F}_{\mathrm{C}}$ was created by Gibson Assembly of three fragments: (1) the vector backbone from a NheI-XhoI $7.168 \mathrm{~kb}$ fragment of pWT35, (2) ACE2 from a $1.86 \mathrm{~kb}$ PCR product of WH1043 and WH1044 amplification of gene-synthesized ACE2 codon optimized for expression in CHO epithelial cell line (AO615ACE2), and (3) $\mathrm{IgG}_{1} \mathrm{~F}_{\mathrm{C}}$ from a $0.701 \mathrm{~kb}$ PCR product of $\mathrm{pXL159}$, using primers WH1045 and WH1046. ACE2 R273Q-IgG $\mathrm{F}_{\mathrm{C}}$ 
was constructed similarly, with the exception that ACE2 R273Q was created by splice by overlap extension (SOE). A $1.86 \mathrm{~kb}$ SOE product was created by amplification with primers WH1043 and WH1044 of two PCR products: (1) 860 bp amplification of AO615ACE2 with primers WH1043 and WH1049, and (2) 1.059 bp amplification product of AO615ACE2 with primers WH1050 and WH1044.

ACE2 T27Y/H34A-IgG $F_{C}$ was constructed by the Gibson Assembly of: (1) a $9.041 \mathrm{~kb}$ NheI-PshA1 digestion fragment of ACE2- $\mathrm{IgG}_{1} \mathrm{~F}_{\mathrm{C}}$ plasmid, and (2) a $0.773 \mathrm{~kb}$ SOE product of primers $5 \mathrm{MutF}$ and $5 \mathrm{MutR}$ of two PCR products. The first PCR product is a $0.154 \mathrm{~kb}$ amplification of plasmid SR9 with primers $5 \mathrm{MutF}$ and ACE2T27YR). The second PCR product is a $0.642 \mathrm{~kb}$ amplification of plasmid SR9 with primers ACE2T27YF and 5MutR.

Most of the triple mutants were created by Gibson Assembly of three fragments: (1) the vector backbone from a $7.168 \mathrm{~kb}$ NheI-XhoI fragment of pWT35, (2) $\mathrm{IgG}_{1} \mathrm{~F}_{\mathrm{C}}$ from a $0.701 \mathrm{~kb}$ PCR amplification of pXL159 with primers WH1045 and WH1046, and (3) the ACE2 variant from a 1.86 bp PCR product containing the three mutations. For the latter, the mutants were amplified with primers WH1043 and WH1044 with templates pWH230 (for T27Y/H34A/R273K), pWH231 (T27Y/H34A/R273L), pWH236 (T27Y/H34A/H345A), pWH233. (T27Y/H34A/ H505L), pWH234 (T27Y/H34A/H374N), and pWH235 (T27Y/H34A/H378N).

ACE2 T27Y/H34A/R273Q was constructed by ligating the 9.041 bp NheI-PshA1 fragment of ACE2 R273Q$\mathrm{IgG}_{1} \mathrm{~F}_{\mathrm{C}}$ and the $0.661 \mathrm{~kb}$ NheI-PshA1 fragment of ACE2 T27Y/H34A-IgG $\mathrm{F}_{\mathrm{C}}$.

Primers $\left(5^{\prime} \rightarrow 3^{\prime}\right)$ :

$\begin{array}{ll}\text { 5MutF } & \text { GTCTTTTCTGCAGTCACCGTCACCGTCCTTG } \\ \text { 5MutR } & \text { TGCGTGAAGATGCTCATAGAGTGGTTTT. } \\ \text { ACE2T27YF } & \text { CGAGGAGCAGGCTAAATACTTTCTGGATAAGTTTAACC } \\ \text { ACE2T27YR } & \text { GGTTAAACTTATCCAGAAAGTATTTAGCCTGCTCCTCG } \\ \text { WH1043 } & \text { CCGTCCTTGACACGAAGCTGCTAGCGCCACCATGAGCAGCAGTAGTTGGCT } \\ \text { WH1044 } & \text { GGTGGGCAAGTATGTGTTTTGTTGCATAGGGAGACCAGTCTG } \\ \text { WH1045 } & \text { AAAACACATACTTGCCCACCTTGTCCTG } \\ \text { WH1046 } & \text { AGTTCTAGAATCGGTATCGCTCATTTGCCAGGGCTCAGTGACAGACTC } \\ \text { WH1049 } & \text { TGGTCCAGAACTGTCCCCACATG } \\ \text { WH1050 } & \text { CATGTGGGGACAGTTCTGGACCA }\end{array}$

Maxcyte transient transfection. For transient expression of ACE2 decoys by Maxcyte transfection, CHO-S cells were cultured in suspension in CD-CHO media supplemented with $8 \mathrm{mM}$-glutamine in shaker flasks at $37{ }^{\circ} \mathrm{C}$ with $125 \mathrm{rpm}$ rotation and $8 \% \mathrm{CO}_{2}$. For transfection, cells in the exponential growth stage were pelleted by centrifugation at $1400 \mathrm{rpm}$ for $10 \mathrm{~min}$, re-suspended in $10 \mathrm{~mL}$ of electroporation buffer, and re-pelleted at $1400 \mathrm{rpm}$ for $5 \mathrm{~min}$. The cell pellet was resuspended at a density of $2 \times 10^{8}$ cells $/ \mathrm{mL}$ in electroporation buffer, mixed with the plasmid harboring either the ACE2(WT)-IgG1Fc or ACE2(WT)-IgA sequence at a concentration of $150 \mu \mathrm{g} / \mathrm{mL}$, and transfected using OC-400 processing assemblies in a Maxcyte ExPERT ATx Transfection System. Transfected cells were incubated for $30 \mathrm{~min}$ at $37^{\circ} \mathrm{C}, 5 \% \mathrm{CO}_{2}$ and then resuspended in Efficient Feed $\mathrm{A}^{\circ}$ Cocktail (CHO-CD EfficientFeed ${ }^{\mathrm{m}} \mathrm{A}+0.2 \%$ Pluronic F- $68+1 \%$ HT Supplement $+1 \%$ L-glutamine) at a density of $\sim 4-6 \times 10^{6}$ cells $/ \mathrm{mL}$. This cell culture was incubated at $37{ }^{\circ} \mathrm{C}$ with $5 \% \mathrm{CO}_{2}$ and $125 \mathrm{rpm}$ rotation overnight, $1 \mathrm{mM}$ sodium buryrate was added, and the culture was further incubated at $32{ }^{\circ} \mathrm{C}$ with $3 \% \mathrm{CO}_{2}$ and $125 \mathrm{rpm}$ for 13 more days; during this incubation period, Maxcyte Feed Cocktail (13.9\% CD Hydrolysate, 69.5\% CHO CD EfficientFeed $^{\text {tw }}$ A, 6.2\% Glucose, 6.9\% FunctionMax ${ }^{\text {ma }}$ Titer Enhancer, 3.5\% L-glutamine) was added at $10 \%$ of the culture volume on Days 3 and 8.

FectoPRO ${ }^{\circ}$ transient transfection of ACE2 Mutant Decoys. For transient expression of ACE2 mutant decoys by FectoPRO ${ }^{\circ}$ transfection, $\mathrm{CHO}-\mathrm{S}$ cells in suspension were cultured in $\mathrm{CD}-\mathrm{CHO}$ media supplemented with $8 \mathrm{mM}$ L-glutamine in shaker flasks at $37{ }^{\circ} \mathrm{C}$ with $125 \mathrm{rpm}$ rotation and $8 \% \mathrm{CO}_{2}$. One day before transfection, $\mathrm{CHO}-\mathrm{S}$ cells were seeded at a density of $1 \times 10^{6}$ cells $/ \mathrm{mL}$ in $45 \mathrm{~mL}$ culture flask. On the day of transfection, $75 \mu \mathrm{L}$ of FectoPRO $^{\circ}$ transfection reagent (PolyPlus-transfection ${ }^{\circ}$ ) was mixed with $5 \mathrm{~mL}$ of $15 \mu \mathrm{g} / \mathrm{mL}$ pcDNA3 plasmid DNA in CD-CHO media and incubated for $10 \mathrm{~min}$ at room temperature. The DNA/transfection reagent mixture was added to $45 \mathrm{~mL}$ of $\mathrm{CHO}-\mathrm{S}$ culture and incubated at $37{ }^{\circ} \mathrm{C}$ with $5 \% \mathrm{CO}_{2}$ and $125 \mathrm{rpm}$ rotation. On Day 3, $50 \mathrm{~mL}$ of the $\mathrm{CD}$-CHO media supplemented with $8 \mathrm{mM} \mathrm{L}$-glutamine was added and the culture incubated for an additional 4 days.

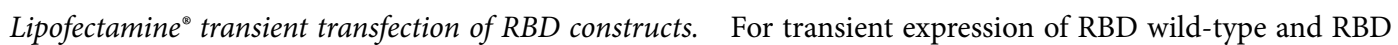
mutants, HEK-293 T cells were cultured and incubated at $37{ }^{\circ} \mathrm{C}$ with $5 \% \mathrm{CO}_{2}$. Plasmids harboring RBD constructs were mixed with lipofectamine with 1:1 (v:v) and incubated for $20 \mathrm{~min}$ at room temperature. The mixture was then added to cultures and incubated for 3-4 days.

Purification of ACE2 Decoy IgGs. The MaxCyte or FectoPRO transfection cell culture medium was centrifuged and filtered through a $0.22 \mu \mathrm{m}$ filter to remove cells and debris, then loaded onto a HiTrap ${ }^{\mathrm{Tx}}$ MabSelect $\mathrm{SuRe}^{\mathrm{Tm}}$ column on the AKTA Pure system pre-equilibrated with $10 \mathrm{mM} \mathrm{Na}$ Phosphate and $150 \mathrm{mM} \mathrm{NaCl}$ at $\mathrm{pH}$ 7.0. After loading, the column was washed with ten column volumes of the same buffer. The protein was eluted with $100 \mathrm{mM}$ sodium acetate, $\mathrm{pH}$ 3.6, then immediately neutralized using $2 \mathrm{M}$ Tris $\mathrm{pH}$ 8.0. The elution fractions were pooled and dialyzed into $10 \mathrm{mM}$ HEPES and $150 \mathrm{mM}$ sodium chloride at $\mathrm{pH}$ 7.4. 
Purification of ACE2 Decoy IgAs. The MaxCyte transfection cell culture medium was centrifuged and filtered through a $0.22 \mu \mathrm{m}$ filter to remove cells and debris, then loaded to a gravity column packed with CaptureSelect ${ }^{\circ}$ IgA resins (Thermo Fisher) pre-equilibrated with $10 \mathrm{mM} \mathrm{Na}$ Phosphate and $150 \mathrm{mM} \mathrm{NaCl}$ at pH 7.0. After loading, the column was washed with ten column volumes of the same buffer. The protein was eluted with $100 \mathrm{mM}$ sodium acetate, $\mathrm{pH} 3.0$, then immediately neutralized using $2 \mathrm{M}$ Tris, $\mathrm{pH}$ 8.0. The elution fractions were pooled and dialyzed into $10 \mathrm{mM}$ Hepes and $150 \mathrm{mM}$ sodium chloride, $\mathrm{pH}$ 7.4.

Purification of RBD and RBD mutants. The Lipofectamine transfection cell culture medium was centrifuged and filtered through a $0.22 \mu \mathrm{m}$ filter to remove cells and debris. A buffer of $50 \mathrm{mM}$ Tris, $100 \mathrm{mM}$ sodium chloride, and $10 \mathrm{mM}$ imidazole was added to the supernatant then loaded to a gravity column packed with Ni-NTA resins (Qiagen) pre-equilibrated with $20 \mathrm{mM}$ Tris, $300 \mathrm{mM}$ sodium chloride, and $10 \mathrm{mM}$ imidazole, pH8.0. After loading, the column was washed with 10 column volumes of the same buffer. The protein was eluted with $20 \mathrm{mM}$ Tris, $150 \mathrm{mM}$ sodium chloride, and $300 \mathrm{mM}$ imidazole. The elution fractions were pooled and dialyzed into $10 \mathrm{mM}$ HEPES and $150 \mathrm{mM}$ sodium chloride, $\mathrm{pH}$ 7.4.

RBD affinity determination of ACE2 decoys by bio-layer interferometry (BLI). The running buffer in all experiments was $10 \mathrm{mM}$ HEPES, $150 \mathrm{mM} \mathrm{NaCl}, \mathrm{pH} 7.4$, with $0.02 \%$ tween 20, and $0.1 \%$ BSA unless otherwise indicated. For the determination of 1:1 binding affinity of ACE2 Decoys against SARS-CoV2 RBD wild-type and mutants, ACE2 Decoys were immobilized on an AHC sensor (Sartorius Corporation) and an RBD concentration series of $200,100,50,25,12.5,6.25,3.125 \mathrm{nM}$ was used to determine the dissociation coefficient $\left(\mathrm{K}_{\mathrm{D}}\right)$. For determining ACE2 Decoy binding affinity with avidity, biotinylated RBD was immobilized on streptavidin (SA) or high-precision SA (SAX) sensors, and the ACE2 Decoy concentration series of 200, 100, 50, 25, 12.5, 6.25, $3.125 \mathrm{nM}$ was used to determine $\mathrm{K}_{\mathrm{D}}$.

Live virus neutralization assay. All aspects of the assay utilizing virus were performed in a BSL3 containment facility according to the ISMMS Conventional Biocontainment Facility SOPs for SARS-CoV-2 cell culture studies. Vero e6 kidney epithelial cells from Cercopithecus aethiops (ATCC CRL-1586) were seeded into 96-well plates at 20,000 cells/well and cultured overnight at $37^{\circ} \mathrm{C}$. The next day, threefold serial dilutions of decoys were prepared in vDMEM ( $2 \%$ FBS, $1 \%$ NEAAs, $1 \%$ Pen-Strep). SARS-CoV-2 virus stock was prepared in vDMEM at 10,000 TCID50/mL, added to the decoy dilutions with $1: 1(\mathrm{v}: \mathrm{v})$ ratio, and incubated for $30 \mathrm{~min}$ at $37^{\circ} \mathrm{C}$. Media was removed from the Vero E6 cells and decoy-virus complexes added then incubated at $37^{\circ} \mathrm{C}$ for $48 \mathrm{~h}$. Cells were fixed with $4 \%$ PFA for $24 \mathrm{~h}$ and stained for nucleocapsid protein to measure infection by percent neutralization. Each well received $60 \mu \mathrm{L}$ of virus or an infectious dose of 600 TCID50. Control wells including six wells on each plate for no virus and virus-only controls were used. The percent neutralization was calculated as $100-\left((\right.$ sample of interest - [average of "no virus"] $) /[$ average of "virus only"] $\left.){ }^{\star} 100\right)$ with a stain for CoV-2 Np imaged on a Celigo Imaging Cytometer (Nexcelom Bioscience).

cPass $^{\mathrm{T} 44}$ surrogate SARS-CoV-2 neutralization assay. High BIND 96-well ELISA plates (Corning \#3369) were coated with $50 \mathrm{ng} /$ well ACE2 wild type decoy overnight at $4{ }^{\circ} \mathrm{C}$. After the antigen solution was removed, each well was blocked with $150 \mu \mathrm{L}$ of $5 \%$ BSA/PBS for 1-2 h at room temperature with shaking. During the blocking step, $40 \mu \mathrm{L}$ of $50 \mathrm{nM}$ RBD and RBD variants were mixed with $40 \mu \mathrm{L}$ of $25 \mu \mathrm{g} / \mathrm{mL}$ of ACE2 decoy were mixed in a 96-well plate and incubated at room temperature for $30 \mathrm{~min}$ with shaking. After blocking, the plate was then washed three times with $250 \mu \mathrm{L}$ of PBS with $0.05 \%$ Tween 20 (PBS-T). To each well, $30 \mu \mathrm{L}$ of 1:1667 diluted mouse anti-His, HRP and $60 \mu \mathrm{L}$ of RBD/ACE2 decoy (or a no decoy control) were added and incubated at room temperature for $30 \mathrm{~min}$. The plated was washed once with $250 \mu \mathrm{L}$ of PBS-T. To develop the signal, $50 \mu \mathrm{L}$ of TMB solution was added and incubated at room temperature in dark for $30 \mathrm{~min}$, followed by addition of $50 \mu \mathrm{L}$ of $2 \mathrm{M}$ sulfuric acid; absorbance was the read at $450 \mathrm{~nm}$. The percent inhibition was calculated using $(1-\mathrm{A} 450$ $(\mathrm{RBD}+$ Decoy)/A450 (RBD only) $) \times 100$.

Assay for ACE2 enzymatic activity. Enzymatic activity ACE2 decoys expressing a variety of mutations-R273Q, R273K, R273L, H245A, H505L, H374N, and H378N-selected to inhibit activity in combination with the S RBD affinity-enhancing mutations T27Y and H34A were assessed in the FRET based ACE2 activity assay.

Received: 25 March 2021; Accepted: 26 May 2021

Published online: 17 June 2021

\section{References}

1. Davies, N.G., Abbott, S., Barnard, R.C., Jarvis, C.I., Kucharski, A.J., Munday, J., Pearson, C.A.B., Russell, T.W., Tully, D.C., Washburne, A.D., Gimma, A. et al. Estimated transmissibility and impact of SARS-CoV-2 lineage B.1.1.7 in England. Science 372(6538), eabg3055 (2021).

2. Plante, J.A., Liu, Y., Liu, J., Xia, H., Johnson, B.A., Lokugamage, K.G., Zhang, X., Muruato, A.E., Zou, J., Fontes-Garfias, C.R. et al. Spike mutation D614G alters SARS-CoV-2 fitness. Nature 592, 116-121 (2021).

3. Hodcroft, E.B., Domman, D.B., Snyder, D.J., Oguntuyo, Van Diest, M.V., Densmore, K.H., Schwalm, K.C., Femling, J., Carroll, J.L., Scott, R.S. et al. Emergence in late 2020 of multiple lineages of SARS-CoV-2 Spike protein variants affecting amino acid position 677. medRxiv 2021.02.12.21251658 (2021).

4. Weisblum, Y., Schmidt, F., Zhang, F., DaSilva, J., Poston, D., Lorenzi, J.C., Muecksch, F., Rutkowska, M., Hoffman, H-H., Michailidis, E. et al. Escape from neutralizing antibodies by SARS-CoV-2 spike protein variants. Elife 9, e61312 (2020). 
5. Garcia-Beltran, W.F., Lam, E.C., St. Denis, K.S., Nitido, A.D., Garcia, Z.H., Hauser, B.M., Feldman, J., Pavlovic, M.N., Gregory, D.J., Poznansky, M.C. et al. Multiple SARS-CoV-2 variants escape neutralization by vaccine-induced humoral immunity. Cell 184(9), 2372-2383 (2021).

6. Cele, S., Gazy, I., Jackson, L., Hwa, S.-H., Tegally, H., Lustig, G., Giandhari, J., Pillay, S., Wilkinson, E., Naidoo, Y. et al. Escape of SARS-CoV-2 501Y.V2 variants from neutralization by convalescent plasma. Nature 593(7857), 142-146 (2021).

7. Zhou, D., Dejnirattisai, W., Supasa, P., Liu, C., Mentzer, A.J., Ginn, H.M., Zhao, Y., Duyvesteyn, H.M.E., Tuekprakhon, A., Nutalai, R. et al. Evidence of escape of SARS-CoV-2 variant B.1.351 from natural and vaccine induced sera. Cell 184, 2348-2361 (2021).

8. Wibmer, C.K., Ayres, F., Hermanus, T., Madzivhandila, M., Kgagudi, P., Oosthuysen, B., Lambson, B.E., de Oliveira, T., Vermeulen, M., van der Berg, K. et al. SARS-CoV-2 501Y.V2 escapes neutralization by South African COVID-19 donor plasma. Nat. Med. 27(4), 622-625 (2021).

9. Chen, R.E., Zhang, X., Case, J.B., Winkler, E.S., Liu, Y., VanBlargan, L.A., Liu, J., Errico, J.M., Xie, X., Suryadevara, N. et al. Resistance of SARS-CoV-2 variants to neutralization by monoclonal and serum-derived polyclonal antibodies. Nat. Med. 27(4), 717-726 (2021).

10. Deng, X., Garcia-Knight, M.A., Khalid, M.M., Servellita, V., Wang, C., Morris, M.K., Sotomayor-González, A., Glasner, D.R., Reyes, K.R., Gliwa, A.S. et al. Transmission, infectivity, and neutralization of a spike L452R SARS-CoV-2 variant. Cell S0092-8674, 00505 (2021).

11. Yu, F., Xiang, R., Deng, X., Wang, L., Yu, Z., Tian, S., Liang, R., Li, Y., Ying, T., \& Jiang, S. Receptor-binding domain-specific human neutralizing monoclonal antibodies against SARS-CoV and SARS-CoV-2. Signal Transduct. Target. Ther. 5(1), 212-223 (2020).

12. Pinto, D., Park, Y-J., Beltramello, M., Walls, A.C., Tortorici, M.A., Bianchi, S., Jaconi, S., Culap, K., Zatta, F., De Marco, A. et al. Cross-neutralization of SARS-CoV-2 by a human monoclonal SARS-CoV antibody. Nature 583(7815), 290-295 (2020).

13. Wang, Z., Schmidt, F., Weisblum, Y., Muecksch, F., Barnes, C.O., Finkin, S., Schaefer-Babajew, D., Cipolla, M., Gaebler, C., Lieberman, J.A. et al. mRNA vaccine-elicited antibodies to SARS-CoV-2 and circulating variants. Nature 592, 616-622 (2021).

14. Fratev, F. The N501Y and K417N mutations in the spike protein of SARS-CoV-2 alter the interactions with both hACE2 and human derived antibody: A Free energy of perturbation study. bioRxiv 2020.12.23.424283 (2020).

15. Simmons, G., Reeves, J.D., Rennekamp, A.J., Amberg, S.M., Piefer, A.J., \& Bates, P. Characterization of severe acute respiratory syndrome-associated coronavirus (SARS-CoV) spike glycoprotein-mediated viral entry. Proc. Natl. Acad. Sci. USA 101(12), 4240-4245 (2004).

16. Li, F. Structure, function, and evolution of coronavirus spike proteins. Annu. Rev. Virol. 3(1), 237-261 (2016).

17. Hoffmann, M., Kleine-Weber, H., Schroeder, S., Kruger, N., Herrler, T., Erichsen, S., Schiergens, T.S., Herrler, G., Wu, N.H., Nitsche, A. et al. SARS-CoV-2 cell entry depends on ACE2 and TMPRSS2 and is blocked by a clinically proven protease inhibitor. Cell 181(2), 271-280.e278 (2020).

18. Benton, D. J., Wrobel, A.G., Xu, P., Roustan, C., Martin, S.R., Rosenthal, P.B., Skehel, J.J., \& Gamblin, S.J. Receptor binding and priming of the spike protein of SARS-CoV-2 for membrane fusion. Nature 588(7837), 327-330 (2020).

19. Greaney, A. J., Starr, T.N., Gilchuk, P., Zost, S.J., Binshtein, E., Loes, A.N., Hilton, S.K., Huddleston, J., Eguia, R. Crawford, K.H.D. et al. Complete mapping of mutations to the SARS-CoV-2 spike receptor-binding domain that escape antibody recognition. Cell Host Microbe S1931-3128, 30624-30627 (2020).

20. Andreano, E., Piccini, G., Licastro, D., Casalino, L., Johnson, N.V., Paciello, I., Monego, S.D., Pantano, E., Manganaro, N., Manenti, A. et al. SARS-CoV-2 escape in vitro from a highly neutralizing COVID-19 convalescent plasma. bioRxiv 2020.12.28.424451 (2020).

21. Chan, K.K., Dorosky, D., Sharma, P., Abbasi, S.A., Dye, J.M., Kranz, D.M., Herbert, A.S., \& Procko, E. Engineering human ACE2 to optimize binding to the spike protein of SARS coronavirus 2. Science 369(6508), 1261-1265 (2020).

22. Inal, J. M. Decoy ACE2-expressing extracellular vesicles that competitively bind SARS-CoV-2 as a possible COVID-19 therapy. Clin. Sci. (Lond.) 134(12), 1301-1304 (2020).

23. Linsky, T.W., Vergara, R., Codina, N., Nelson, J.W., Walker, M.J., Su, W., Barnes, C.O., Hsiang, T-Y., Esser-Nobis, K., Yu, K., et al. De novo design of potent and resilient hACE2 decoys to neutralize SARS-CoV-2. Science 370(6521), 1208-1214 (2020).

24. Glasgow, A., Glasgow, J., Limonta, D., Solomon, P., Lui, I., Zhang, Y., Nix, M.A., Rettko, N.J., Zha, S., Yamin, R., et al. Engineered ACE2 receptor traps potently neutralize SARS-CoV-2. Proc. Natl. Acad. Sci. 117(45), 28046-28055 (2020).

25. Batlle, D., Wysocki, J. \& Satchell, K. Soluble angiotensin-converting enzyme 2: A potential approach for coronavirus infection therapy? Clin. Sci. (Lond.) 134(5), 543-545 (2020).

26. Sokolowska, M. Outsmarting SARS-CoV-2 by empowering a decoy ACE2. Signal Transduct. Target. Ther. 5(1), 260-262 (2020).

27. Monteil, V., Kwon, H., Prado, P., Hagelkruys, A., Wimmer, R.A., Stahl, M., Leopoldi, A., Garetta, E., Hurtado Del Pozo, C., Prosper, F., et al. Inhibition of SARS-CoV-2 infections in engineered human tissues using clinical-grade soluble human ACE2. Cell 181(4), 905-913.e907 (2020).

28. Nelson, G., Buzko, O., Bassett, A., Spilman, P., Niazi, K., Rabizadeh, S., \& Soon-Shiong, P. Millisecond-scale molecular dynamics simulation of spike RBD structure reveals evolutionary adaption of SARS-CoV-2 to stably bind ACE2. bioRxiv 2020.12.11.422055 (2020).

29. Walls, A.C., Park, Y.J., Tortorici, M.A., Wall, A., McGuire, A.T., \& Veesler, D. Structure, function, and antigenicity of the SARSCoV-2 spike glycoprotein. Cell 181(2), 281-292.e286 (2020).

30. Wrapp, D., Wang, N., Corbett, K.S., Goldsmith, J.A., Hsieh, C-L., Abiona, O., Graham, B.S., \& McLellan, J.S. Cryo-EM structure of the 2019-nCoV spike in the prefusion conformation. Science 367(6483), 1260-1263 (2020).

31. Tegally, H., Wilkinson, E., Lessells, R.J., Giandhari, J., Pillay, S., Msomi, N., Mlisana, K., Bhiman, J.N., von Gottberg, A., Walaza, S. et al. Sixteen novel lineages of SARS-CoV-2 in South Africa. Nat. Med. 27, 440-446 (2021).

32. Leung, K., Shum, M.H., Leung, G.M., Lam, T.T., \& Wu, J.T. Early transmissibility assessment of the N501Y mutant strains of SARS-CoV-2 in the United Kingdom, October to November 2020. Euro. Surveill. 26(1), 2002106-2002112 (2021).

33. Zhang, W., Davis, B.D., Chen, S.S., Sincuir Martinez, J.M., Plummer, J.T., \& Vail, E. Emergence of a novel SARS-CoV-2 variant in Southern California. JAMA 325, 1324-1316 (2021)

34. McCallum, M., Bassi, J., Marco, A., Chen, A., Walls, A.C., Iulio, J.D., Tortorici, M.A., Navarro, M.J., Silacci-Fregni, C., Saliba, C., et al. SARS-CoV-2 immune evasion by variant B.1.427/B.1.429. bioRxiv 2021.03.31.437925 (2021).

35. Tchesnokova, V., Kulakesara, H., Larson, L., Bowers, V., Rechkina, E., Kisiela, D., Sledneva, Y., Choudhury, D., Maslova, I., Deng, K. et al. Acquisition of the L452R mutation in the ACE2-binding interface of Spike protein triggers recent massive expansion of SARS-Cov-2 variants. bioRxiv 2021.02.22.432189 (2021).

36. Liu, P., Xie, X., Gao, L. \& Jin, J. Designed variants of ACE2-Fc that decouple anti-SARS-CoV-2 activities from unwanted cardiovascular effects. Int. J. Biol. Macromol. 165(Pt B), 1626-1633 (2020).

37. Warner, F.J., Lew, R.A., Smith, A.I., Lambert, D.W., Hooper, N.M., \& Turner, A.J. Angiotensin-converting enzyme 2 (ACE2), but not ACE, is preferentially localized to the apical surface of polarized kidney cells. J. Biol. Chem. 280(47), 39353-39362 (2005).

38. Kuba, K., Imai, Y., Rao, S., Gao, H., Guo, F., Guan, B., Huan, Y., Yang, P., Zhang, Y., Deng, W. et al. A crucial role of angiotensin converting enzyme 2 (ACE2) in SARS coronavirus-induced lung injury. Nat. Med. 11(8), 875-879 (2005).

39. Alifano, M., Alifano, P., Forgez, P., \& Iannelli, A. Renin-angiotensin system at the heart of COVID-19 pandemic. Biochimie 174, 30-33 (2020).

40. Kramkowski, K., Mogielnicki, A., Leszczynska, A. \& Buczko, W. Angiotensin-(1-9), the product of angiotensin I conversion in platelets, enhances arterial thrombosis in rats. J. Physiol. Pharmacol. 61(3), 317-324 (2010). 
41. Guy, J. L., Jackson, R. M., Jensen, H. A., Hooper, N. M. \& Turner, A. J. Identification of critical active-site residues in angiotensinconverting enzyme-2 (ACE2) by site-directed mutagenesis. FEBS J. 272(14), 3512-3520 (2005).

42. Li, W., Moore, M.J., Vasilieva, N., Sui, J., Wong, S.K., Berne, M.A., Somasundaran, M., Sullivan, J.L., Luzuriaga, K., Greenough, T.C. et al. Angiotensin-converting enzyme 2 is a functional receptor for the SARS coronavirus. Nature 426(6965), 450-454 (2003).

43. Ramanathan, M., Ferguson, I.D., Miao, W., \& Khavari, P.A. SARS-CoV-2 B.1.1.7 and B.1.351 spike variants bind human ACE2 with increased affinity. Lancet Infect. Dis. S1473-3099, 002660-62 (2021).

44. Tan, C.W., Chia, W.N., Qin, X., Liu, P., Chen, M.I., Tiu, C., Hu, Z., Chen, V.C., Young, B.E., Sia, W.R. et al. A SARS-CoV-2 surrogate virus neutralization test based on antibody-mediated blockage of ACE2-spike protein-protein interaction. Nat. Biotechnol. 38(9), 1073-1078 (2020).

45. Babin, V., Roland, C. \& Sagui, C. Adaptively biased molecular dynamics for free energy calculations. J. Chem. Phys. 128(13), 134101 (2008).

46. Karim, S.S.A. Vaccines and SARS-CoV-2 variants: the urgent need for a correlate of protection. Lancet 397, 1263-1264 (2021).

47. Madhi, S.A., Baillie, V., Cutland, C.L., Voysey, M., Koen, A.L., Fairlie, L., Padayachee, S.D., Dheda, K., Barnabas, S.L., Bhorat, Q.E. et al. Efficacy of the ChAdOx1 nCoV-19 Covid-19 Vaccine against the B.1.351 Variant. N. Engl. J. Med. 384, 1885-1898 (2021).

48. Edara, V.V., Norwood, C., Floyd, K., Lai, L., Davis-Gardner, M.E., Hudson, W.H., Mantus, G., Nyhoff, L.E., Adelman, M.W., Fineman, R. et al. Reduced binding and neutralization of infection- and vaccine-induced antibodies to the B.1.351 (South African) SARS-CoV-2 variant. bioRxiv 2021.02.20.43046 (2021).

49. Wang, P., Nair, M.S., Liu, L., Iketani, S., Luo, Y., Guo, Y., Wang, M., Yu, J., Zhang, B., Kwong, P.D. et al. Antibody resistance of SARS-CoV-2 variants B.1.351 and B.1.1.7. Nature 593, 130-135 (2021).

50. Geers, D., Shamier, M.C., Bogers, S., den Hartog, G., Gommers, L., Nieuwkoop, N.N., Schmitz, K.S., Rijsbergen, L.C., van Osch, J.A.T, Dijkhuizen, E. et al. SARS-CoV-2 variants of concern partially escape humoral but not T-cell responses in COVID-19 convalescent donors and vaccinees. Sci. Immunol. 6(59), eabj1750 (2021).

51. Huang, K.Y., Lin, M.S., Kuo, T.C., Chen, C.L., Lin, C.C., Chou Y.C., Chao, T.L., Pang, Y.H., Kao, H.C., Huang, R.S. et al. Humanized COVID-19 decoy antibody effectively blocks viral entry and prevents SARS-CoV-2 infection. EMBO Mol. Med. 13(1), e12828 (2020).

52. Nelson, G., Buzko, O., Spilman, P., Niazi, K., Rabizadeh, S., Soon-Shiong, P. Molecular dynamic simulation reveals E484K mutation enhances spike RBD-ACE2 affinity and the combination of E484K, K417N and N501Y mutations (501Y.V2 variant) induces conformational change greater than N501Y mutant alone, potentially resulting in an escape mutant. bioRxiv 2021.01.13.426558 (2021).

53. Ameratunga, R., Lehnert, K., Leung, E., Comoletti, D., Snell, R., Woon, S.T., Abbott, W., Mears, E., Steele, R., McKee, J. et al. Inhaled modified angiotensin converting enzyme 2 (ACE2) as a decoy to mitigate SARS-CoV-2 infection. N. Z. Med. J. 133(1515), 112-118 (2020).

54. Cocozza, F., Nevo, N., Piovesana, E., LaHaye, X., Buchrieser, J., Schwartz, O., Manel, N., Tkach, M.,Thery, C., \& Martin-Jauler, L. Extracellular vesicles containing ACE2 efficiently prevent infection by SARS-CoV-2 Spike protein-containing virus. J. Extracell. Vesicles 10(2), e12050 (2020).

55. Rao, L., Xia, S., Xu, W., Tian, R., Yu, G., Gu, C., Pan, P., Meng, Q-F., Cai, X., Qu, D. et al. Decoy nanoparticles protect against COVID-19 by concurrently adsorbing viruses and inflammatory cytokines. Proc. Natl. Acad. Sci. 117(44), 27141 (2020).

56. Kim, J., Mukherjee, A., Nelson, D., Jozic, A., \& Sahay, G. Rapid generation of circulating and mucosal decoy ACE2 using mRNA nanotherapeutics for the potential treatment of SARS-CoV-2. bioRxiv 2020.07.24.205583 (2020).

57. Wang, Z., Lorenzi, J.C.C., Muecksch, F., Finkin, S., Viant, C., Gaebler, C., Cipolla, M., Hoffman, H-H., Oliveira, T.Y., Oren, D.A. et al. Enhanced SARS-CoV-2 neutralization by dimeric IgA. Sci. Transl. Med. 13(577), eabf1555 (2021).

58. Rice, A., Verma, M., Shin, A., Zakin, L., Sieling, P., Tanaka, S., Adisetiyo, H., Taft, J., Patel, R., Buta, S., et al. A next generation bivalent human Ad5 COVID-19 vaccine delivering both spike and nucleocapsid antigens elicits Th1 dominant CD4+, CD8+ T-cell and neutralizing antibody responses. bioRxiv https://doi.org/10.1101/2020.07.29.227595 (2020).

59. Gabitzsch, E., Safrit, J.T., Verma, M., Rice, A., Sieling, P., Zakin, L., Shin, A., Morimoto, B., Adisetiyo, H., Wong, R. et al. Complete protection of nasal and lung airways against SARS-CoV-2 challenge by antibody plus Th1 dominant $\mathrm{N}$ - and S-specific T-cell responses to subcutaneous prime and thermally-stable oral boost bivalent hAd5 vaccination in an NHP study. bioRxiv 2020.12.08.416297 (2021).

60. Yan, R., Zhang, Y., Li, Y., Xia, L., Guo, Y. \& Zhou, Q. Structural basis for the recognition of SARS-CoV-2 by full-length human ACE2. Science 367(6485), 1444-1448 (2020).

61. Maier, J. A., Martinez, C., Kasavajhala, K., Wickstrom, L., Hauser, K.E., \& Simmerling, C. ff14SB: Improving the accuracy of protein side chain and backbone parameters from ff99SB. J. Chem. Theory Comput. 11(8), 3696-3713 (2015).

62. Case, D.A., Ben-Shalom, I.Y., Brozell, S.R., Cerutti, D.S., Cheatham III, T.E., Cruzeiro, V.W.D., Darden, T.A., Duke, R.E., Ghoreishi, D., Giambasu, G. et al. AMBER 19. Ambertools 2019, www.ambermd.org.

63. Price, D. J. \& Brooks 3rd, C. L. A modified TIP3P water potential for simulation with Ewald summation. J. Chem. Phys. 121(20), 10096-10103 (2004).

64. Li, P., Song, L. F. \& Merz Jr., K. M. Systematic parameterization of monovalent ions employing the nonbonded model. J. Chem. Theory Comput. 11(4), 1645-1657 (2015).

65. Barducci, A., Bussi, G. \& Parrinello, M. Well-tempered metadynamics: A smoothly converging and tunable free-energy method. Phys. Rev. Lett. 100(2), 020603 (2008).

66. Minoukadeh, K., Chipot, C. \& Lelièvre, T. Potential of mean force calculations: A multiple-walker adaptive biasing force approach. J. Chem. Theory Comput. 6(4), 1008-1017 (2010).

67. Roe, D. R. \& Cheatham 3rd, T. E. PTRAJ and CPPTRAJ: Software for processing and analysis of molecular dynamics trajectory data. J. Chem. Theory Comput. 9(7), 3084-3095 (2013).

\section{Acknowledgements}

We would like to thank Phil Yang of ImmunityBio, Inc. for his coordination of project updates for this study.

\section{Author contributions}

S.T. performed protein purification of ACE2(WT)IgGFc and ACE2(WT)IgAFc, BLI characterization, RBD-SD1, RBD mutant protein production, the cPass assay, Tm analysis, developability assays and co-wrote the manuscript; G.N. performed all MD simulations and co-wrote the manuscript, and GN/OB choose the mutations to be tested in as ACE2 decoys in vitro; C.A.O. cloned of RBD-SD1 and RBD variants and performed T293 transfection; W.H. cloned the ACE2(WT)IgGFc, ACE2(WT)IgAFc, and ACE2 mutant constructs; A.S. was responsible for ACE2 decoy cloning and production; M.G. purified mutant ACE2 decoys; P.S. analyzed and graphed data, created manuscript figures and wrote the manuscript; and K.N., S.R., and P.S.S. initiated the project, guided its direction, reviewed data and edited the manuscript. J.T., R.P., A.R., and S.B. performed the live SARS-CoV-2 virus neutralization assay in the laboratory and under the supervision of D.B. All authors reviewed the manuscript. 


\section{Competing interests}

Authors with an ImmunityBio affiliation including Shiho Tanaka, Gard Nelson, C. Anders Olson, Oleksandr Buzko, Wendy Higashide, Annie Shin, Marcos Gonzalez, Patricia Spilman, Kayvan Niazi, Shahrooz Rabizadeh and Patrick Soon-Shiong designed, created or tested the ACE2 decoy, which may become a commercial product. Authors Justin Taft, Roosheel Patel, Sofija Buta, Ashley Richardson, and Dusan Bogunovic have no competing interests.

\section{Additional information}

Supplementary Information The online version contains supplementary material available at https:/doi.org/ 10.1038/s41598-021-91809-9.

Correspondence and requests for materials should be addressed to S.T.

Reprints and permissions information is available at www.nature.com/reprints.

Publisher's note Springer Nature remains neutral with regard to jurisdictional claims in published maps and institutional affiliations.

(c) (i) Open Access This article is licensed under a Creative Commons Attribution 4.0 International

License, which permits use, sharing, adaptation, distribution and reproduction in any medium or format, as long as you give appropriate credit to the original author(s) and the source, provide a link to the Creative Commons licence, and indicate if changes were made. The images or other third party material in this article are included in the article's Creative Commons licence, unless indicated otherwise in a credit line to the material. If material is not included in the article's Creative Commons licence and your intended use is not permitted by statutory regulation or exceeds the permitted use, you will need to obtain permission directly from the copyright holder. To view a copy of this licence, visit http://creativecommons.org/licenses/by/4.0/.

(C) The Author(s) 2021 